\title{
Activation of the MEF2 transcription factor in skeletal muscles from myotonic mice
}

\author{
Hai Wu and Eric N. Olson \\ Department of Molecular Biology, University of Texas Southwestern Medical Center at Dallas, Dallas, Texas, USA \\ Address correspondence to: Eric N. Olson, Department of Molecular Biology, \\ University of Texas Southwestern Medical Center at Dallas, Dallas, Texas 75390-9148, USA. \\ Phone: (214) 648-1187; Fax: (214) 648-1196; E-mail: eolson@hamon.swmed.edu.
}

Received for publication March 7, 2002, and accepted in revised form April 9, 2002.

\begin{abstract}
Becker syndrome, a recessive nondystrophic myotonia caused by mutations in the chloride channel 1 gene (CLCN1), is characterized by delayed muscle relaxation after contraction. The ADR (arrested development of righting response) mouse is an animal model for Becker syndrome. Skeletal muscles from ADR myotonic animals show an increased number of oxidative fibers with a lack of glycolytic fibers as well as signs of muscle hypertrophy. Through breeding ADR myotonic mice with mice harboring a MEF2-dependent reporter gene, we found that the transcriptional activity of MEF2 was dramatically enhanced in myotonic muscles. Post-translational induction of MEF2 transcriptional activity correlated with the activation of p38 MAPK and did not affect MEF2 DNA-binding affinity. Expression of class II histone deacetylases (HDACs), which repress MEF2-dependent gene expression, was significantly reduced in skeletal muscles from myotonic mice. These findings suggest that the combined effects of class II HDAC deficiency and p38 MAPK activation lead to potent upregulation of MEF2 transcriptional activity, which contributes to the long-term changes in gene expression and fiber-type transformation observed in myotonic skeletal muscles. These findings provide new molecular targets for potential treatment of congenital myotonia.
\end{abstract}

J. Clin. Invest. 109:1327-1333 (2002). doi:10.1172/JCI200215417.

\section{Introduction}

Myotonia is the symptom of several skeletal muscle diseases that are characterized by delayed relaxation of the affected muscle fibers after voluntary contractions (1). The primary defects in most myotonia patients are either sodium channel mutations, as in myotonia fluctuans, myotonia permanens, and acetacolamideresponsive myotonia (2) or chloride channel mutations as in myotonia congenita and myotonia levior $(3,4)$.

Becker syndrome is a recessive nondystrophic form of myotonia congenita, which is caused by mutations in the CLCN1 gene encoding the voltage-dependent chloride channel CLC-1 (5). CLC-1 is expressed almost exclusively in skeletal muscles (6), and the functional chloride channel is most probably a dimer of CLC-1 proteins (7). In recessive myotonia congenita, missense, nonsense, or deletion mutations in the CLCN1 gene result in loss of function (e.g., severe truncation) or changes in voltage-dependence of the chloride channel such that the open probability of the channel reduces at voltages positive to chloride equilibrium potential $(8,9)$. Chloride conductance via CLC- 1 is primarily responsible for maintaining the negative resting membrane potential $(-90 \mathrm{mV})$ of skeletal muscles (10). In myotonia linked to CLCN1 mutations, skeletal muscle cells are hyperexcitable due to the lack of chloride influx (11).

ADR (arrested development of righting response) myotonic mice harbor mutations in the CLCN1 gene and serve as mouse models of human Becker syndrome (12).
These mutant CLCN1 alleles arise either spontaneously or are induced by the 1-ethylnitrosourea mutagen, and they do not complement each other (13). In rodents, the prototypical mutant allele $C L C N 1^{\text {adr }}$ is due to a retroposon insertion (12). The $C L C N 1^{\text {adr-mto }}$ allele involves a nonsense mutation resulting in a severely truncated CLC-1 product (14). Mice with one mutant CLCN1 gene are normal, whereas by 2 weeks of age homozygous mutants display myotonic symptoms of prolonged stiff extension posture of the limbs when they are disturbed. Homozygous mutants also grow more slowly and weigh about $40 \%$ less than their wild-type littermates at adulthood. Interestingly, ADR myotonic mice lack type IIb glycolytic skeletal muscle fibers, but show no sign of muscle hypertrophy (15), which is one of the common features of human myotonic patients (16). Some human patients also show a complete absence of type IIb fibers in their skeletal muscles (17). How chloride channel mutations cause fiber-type switching is not known.

Myocyte enhancer factor-2 (MEF2) transcription factors are a family of muscle-enriched nuclear factors that are critical for muscle differentiation and development (18). Recently, we and others identified MEF2 as an important regulator of muscle hypertrophy and skeletal myofiber diversity, which acts downstream of calcium signaling (19). MEF2 is activated by calcium/calmodulin-dependent enzymes, such as calcineurin and calcium, calmodulin-dependent protein kinases (CaMKs), in response to increases in intracellu- 
lar calcium concentration $\left(\left[\mathrm{Ca}^{2+}\right]_{i}\right)(20,21)$. Calcineurin can dephosphorylate MEF2 and enhance MEF2 transcriptional activity (22), while CaMK signaling results in the dissociation of histone deacetylases (HDACs) from the N-terminal DNA-binding domain of MEF2 and relieves the repression imposed on MEF2 by HDACs $(23,24)$. Stress-responsive mitogen-activated protein kinases (MAPKs), such as p38 and ERK5, also enhance MEF2 activity by phosphorylation of the transcription activation domain of MEF2 $(25,26)$.

In this study, using "MEF2 indicator mice", which harbor a $\beta$-galactosidase transgene controlled by three copies of the MEF2 DNA-binding element (27), we observed that the in vivo transcriptional activity of MEF2 was dramatically induced in myotonic muscles. MEF2 activation occurred through a post-translational mechanism and correlated with the activation of p38 MAPK, a known activator of MEF2. Interestingly, the expression level of class II HDAC (HDAC4, -5, -7) proteins was significantly reduced in skeletal muscles from myotonic mice compared with wild-type mice. We propose that the combined effects of class II HDAC deficiency and p38 MAPK activation lead to potent upregulation of MEF2 transcriptional activity, which contributes to the long-term changes in gene expression observed in myotonic skeletal muscles.

\section{Methods}

Animals. Mice carrying the desMEF2-lacZ transgene (MEF2 indicator mice) were described previously (27). Briefly, after removal of prokaryotic sequences, $\beta$-galactosidase transgenes controlled by three copies of MEF2 DNA-binding elements derived from the desmin promoter were introduced by microinjection into fertilized oocytes of $\mathrm{C} 57 \mathrm{~B} 6 / \mathrm{C} 3 \mathrm{H}$ mice. The resulting transgenic offspring were identified by Southern blot or PCR analysis of genomic DNA. Two lines of ADR myotonic mice were purchased from The Jackson Laboratory (SWR/J-Clcn 1 $1^{\text {adr-mto/+ }}$ and BALB/cByJ-Clcn1 $1^{\text {adr-mto/++ }}$; Bar Harbor, Maine, USA). Both lines have mutations in the CLCN1 gene and display the same myotonic phenotype. All experiments involving animals were reviewed and approved by the Institutional Animal Care and Research Advisory Committee.

$\mathrm{Gel}$ electrophoretic mobility shift assay. A synthetic oligonucleotide representing a high-affinity MEF2binding motif ( $5^{\prime}$ GATCCTCTAAAAATAACCCT3') from the muscle creatine kinase (MCK) gene (28) was annealed to its complementary strand, labeled with polynucleotide kinase and $\gamma-{ }^{32} \mathrm{P}-\mathrm{ATP}(3,000 \mathrm{Ci} / \mathrm{mmol}$; Amersham, Piscataway, New Jersey, USA), and incubated with muscle protein extracts $(10 \mu \mathrm{g})$ in binding buffer (20 mM HEPES, pH 7.6, $50 \mathrm{mM} \mathrm{KCl,} \mathrm{10 \%} \mathrm{glyc-}$ erol, $0.2 \mathrm{mM}$ EDTA, $1 \mathrm{mM}$ DTT, and $1 \mu \mathrm{g}$ of polydI$\mathrm{dC}$ per lane) for 20 minutes at room temperature. Competitive binding assays were conducted under the same conditions, with the addition of 2 pmol (100fold molar excess) of unlabeled competitor oligonucleotides that included the same sequences as the labeled ones. DNA-protein complexes were resolved on $4 \%$ polyacrylamide gels at $4^{\circ} \mathrm{C}$ in $45 \mathrm{mM}$ Tris, $45 \mathrm{mM}$ borate, $1 \mathrm{mM}$ EDTA buffer, dried, and visualized using a Phosphor-Imager system (Molecular Dynamics, Sunnyvale, California, USA).

Immunoblotting. Muscle protein was extracted from dissected white vastus muscles by homogenization in lysis buffer (50 mM HEPES, pH 7.6, $250 \mathrm{mM} \mathrm{NaCl}$, $0.1 \%$ Nonidet P-40 [NP-40], 5 mM EDTA, 1 mM DTT, and protease inhibitor cocktail; Roche Applied Science, Indianapolis, Indiana, USA). Lysate was cleared by centrifugation at $15,000 \mathrm{~g}$ for 10 minutes. The supernatant fractions were then boiled in SDS-PAGE-loading buffer, separated by SDS-PAGE, and transferred to nitrocellulose membranes following standard immunoblotting procedures. Ab's against $\beta$-galactosidase (1:2,000; Promega Corp., Madison, Wisconsin, USA), MEF2A (1:2,000; Santa Cruz Biotechnology Inc., Santa Cruz, California, USA), MEF2C (1:500; gift from John Schwarz, University of Texas Medical School at Houston, Houston, Texas, USA), MEF2D (1:1,000; Transduction Laboratories, Lexington, Kentucky, USA), pan-p38 MAPK (1:1,000; Cell Signaling Technology, Beverly, Massachusetts, USA), phospho-p38 MAP kinase (1:1,000; Cell Signaling Technology), $\alpha$-tubulin (1:3,000; Sigma-Aldrich, St. Louis, Missouri, USA), myoglobin (1:3,000; DAKO Corp., Carpinteria, California, USA), and HDAC1, -2 , and -3 (1:1,000 for all; Sigma-Aldrich), HDAC4, -5, and -7 (1:2,000, 1:500, and 1:1,000, respectively; Cell Signaling Technology) were used for immunoblot analyses.

$R N A$ isolation and RT-PCR. Total RNA was prepared from mouse skeletal muscles using RNA STAT-60 (TelTest Inc., Friendswood, Texas, USA) following the manufacturer's instructions. RNA (1.5 $\mu \mathrm{g}$ total) was converted to cDNA using oligo-dT primer and Superscript II reverse transcriptase (Invitrogen Corp., San Diego, California, USA). For PCR reactions, $2 \%$ of the cDNA pool was amplified. PCR cycles were optimized for each set of primers. Primer sequences are as follows: $\alpha$-actin, forward (fwd): $5^{\prime}$ TTCCTTCGTGACCACAGCTGAACGTG3', reverse (rev): 5'GCAGACTCCATACCGATAAAGGAAG3'; myoglobin, fwd: 5'GCACAACCTTGCAGAGAAAGCCAGGAG3', rev: 5'CAGATCTAACCCCAACCACTTTCCACTG3'; HDAC1, fwd: 5'GGCATCTTAGCAGAGCTCC3', rev: 5'CTGAGCAAGGTCTGCAGC3'; HDAC2, fwd: 5'AAATCAGCTCAGAAAGGCCA3', rev: 5' CCAAGGACAATAGTGGTGAG3'; HDAC3, fwd: 5'TTTGAGTTCTGCTCGCGTTAC3', rev: 5'CAGGTTTTCAAAGATTGTCTGGG3'; HDAC4,fwd: 5'CTTGAGCTGCTGCAGCTTC3', rev: 5'CAGATGGACTTTCTGGCCG3'; HDAC 5, fwd: 5'GAAGCACCTCAAGCAGCAGCAGG3', rev: 5'CACTCTCTTTGCTCTTCTCCTTGTT3'; HDAC6, fwd: 5'TCTCAACTGATCTCTCCAGG3', rev: 5'ACGCTGACTACATTGCTGCT3'; HDAC7, fwd: 5'TCACCATCAGCCTCTGAG3', rev: 5'AGCTGGCTGAAGTGATCC3'; HDAC8, fwd: 5'AACACGGCTCGATGCTGG3'， rev: 5'CCAGCTGCCACTTGATGC3'; HDAC9 (MITR), fwd: 5'TCAGAGGTTCCTATGGGCCTG3', rev: 5'TGGAGACGTTCCACTGAGGG3'; MHCIIa, fwd: 
a

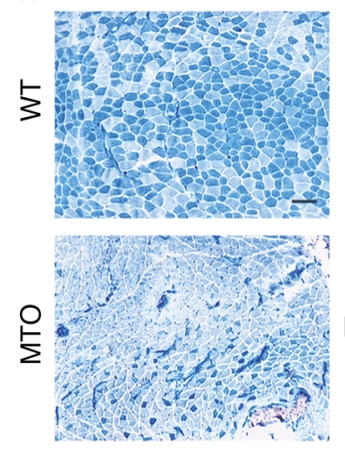

b
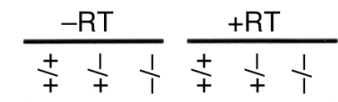

MHC IIa

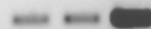

MHC IIb

Myoglobin
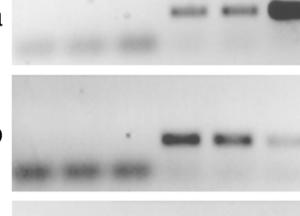

$\alpha$-actin

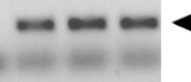

5'ACAAATCTATCCAAGTTCCG3', rev: 5'TTCGGTCATTCCACAGCATC3'; MHCIIb, fwd: 5'CCGTGATATACAGGACAGTG3', rev: 5'GTTCCGTAAGATCCAGCACG3'.

$\beta$-galactosidase staining. Dissected muscles were fixed with $2 \%$ paraformaldehyde/ $0.1 \%$ glutaraldehyde in PBS on ice for 30-45 minutes followed by washing and X-gal staining ( $5 \mathrm{mM}$ ferrocyanide, $5 \mathrm{mM}$ ferricyanide, $2 \mathrm{mM}$ $\mathrm{MgCl}_{2}, 1 \mathrm{mg} / \mathrm{ml} \mathrm{X}$-gal, $0.01 \%$ sodium deoxycholate, and $0.02 \% \mathrm{NP}-40$ ) for $1-12$ hours at room temperature.

Metachromatic fiber typing of skeletal myofibers. Fiber typing was performed with cryostat sections of plantaris (PLA) muscles using the metachromatic dye-ATPase method (29). All steps were carried out at room temperature. Muscle sections were treated in preincubation buffer $\left(50 \mathrm{mM}\right.$ potassium acetate, $\mathrm{pH}$ 4.4, $17 \mathrm{mM} \mathrm{CaCl}_{2}$ ) for 8 minutes, followed with three washes in Tris rinse buffer (300 mM Tris- $\mathrm{HCl}, \mathrm{pH} 7.8,53 \mathrm{mM} \mathrm{CaCl}_{2}$ ). Muscle slides were then put into incubation buffer $(53 \mathrm{mM}$ glycine, $\mathrm{pH}$ 9.4, $28 \mathrm{mM} \mathrm{CaCl}_{2}, 65 \mathrm{mM} \mathrm{NaCl}, 47.5$ $\mathrm{mM} \mathrm{NaOH}, 4 \mathrm{mM}$ ATP) for 25 minutes and rinsed three times in $1 \%$ (wt/vol) $\mathrm{CaCl}_{2}$ solution. Then, $0.1 \%$ toluidine blue was used to stain muscle fibers for 1 minute. The sections were rinsed in $\mathrm{H}_{2} \mathrm{O}$ and dehydrated with ethanol.

\section{Results}

Fiber-type switches (from type IIb to IIa) in skeletal muscles from ADR myotonic mice. Mammalian skeletal myofibers can be categorized into glycolytic and oxidative groups based upon their

\section{Figure 2}

Activation of MEF2 in myotonic skeletal muscles. Soleus (SOL), PLA, EDL, and WV muscles were dissected from the hind limbs of MEF2 indicator mice that also carry different alleles of the CLCN1 gene, including wild-type alleles $(+/+)$ and heterozygous (+/-) and homozygous (-/-) mutant CLCN1 alleles. Muscles were fixed and stained for $\beta$-galactosidase activity. The distribution of $\beta$-galactosidase appeared to be uniform along the myofibers. Note that the MEF2-lacZ transgene is specifically activated in PLA, EDL, and WV muscles of the homozygous mutant $(\times 20$ magnification; scale bar $=1 \mathrm{~mm})$.

\section{Figure 1}

Transformation of glycolytic fibers into oxidative ones in myotonic PLA muscles. (a) Specialized myofiber subtypes assessed by myosin ATPase histochemistry revealed fiber-type switch (type IIb to Ila) in PLA muscles from ADR myotonic mice. Myosin ATPase activity was determined by $\mathrm{pH}$-dependent histochemistry able to distinguish types IIb (blue-stained) and Ila (unstained) in PLA sections from wild-type (WT) and myotonic (MTO) mice $(\times 100$ magnification; scale bar $=100 \mu \mathrm{m})$. (b) Transcripts for MHC Ila, IIb, and myoglobin were detected with RT-PCR using cDNA from WV muscles of wild-type $(+/+)$, heterozygous $(+/-)$, and homozygous $(-/-)$ CLCN1 mutant mice.

metabolic profiles (30). Oxidative fibers primarily rely on oxidative phosphorylation to provide energy, whereas glycolytic fibers primarily use glycolysis to generate ATP. Consequently, oxidative fibers contain more mitochondria, higher concentration of myoglobin, and better blood supply to facilitate oxygen transfer. Different myofibers also express distinct isoforms of contractile proteins, most notably myosin heavy chains (MHCs), which determine the contractile speed of the myofibers (31). In rodents, most glycolytic fibers are type IIb fast fibers because they express MHC IIb isoforms, and oxidative fibers include type I slow and type IIa fast fibers (32). Glycolytic fibers can be transformed into oxidative ones, and vice versa, under different circumstances (33). Patients with Becker syndrome show complete absence of glycolytic fibers in their skeletal muscles (17). ADR myotonic mice display a similar phenotype (15), which is due to a loss-of-function mutation in the CLCN1 gene.

As shown in Figure 1a, PLA muscle from wild-type mice contains $70 \%$ type IIb and 30\% type IIa fibers, while in ADR myotonic mice Type IIa fibers constitute $80 \%$ of the total fibers in plantaris muscle. The

\section{o}
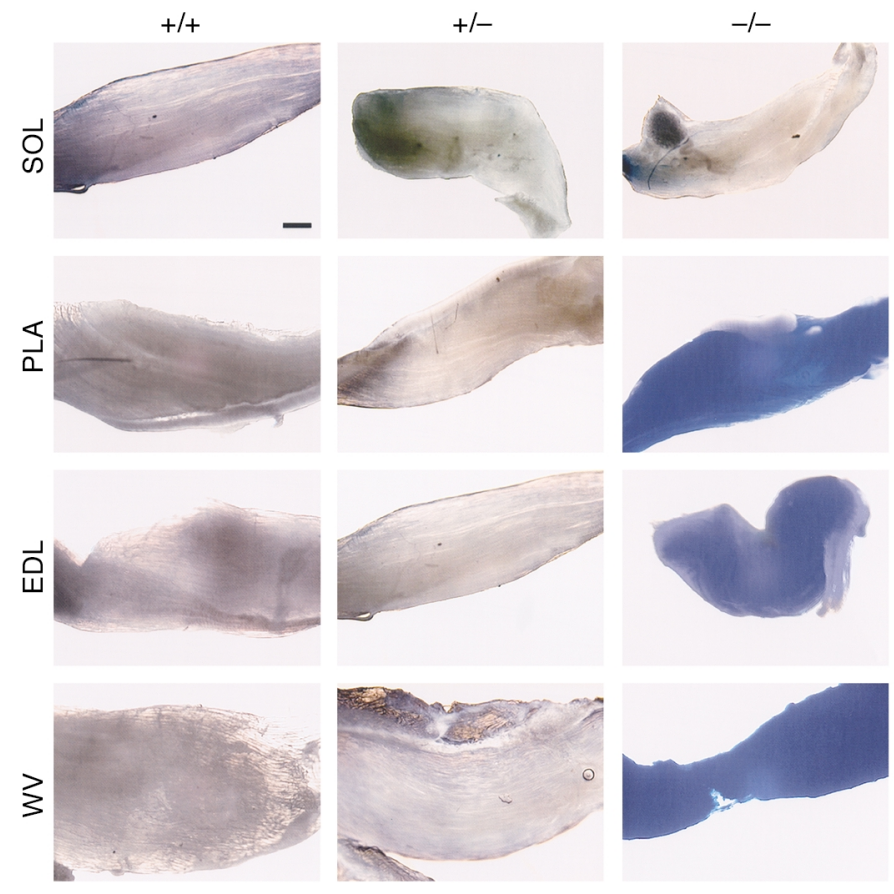
a

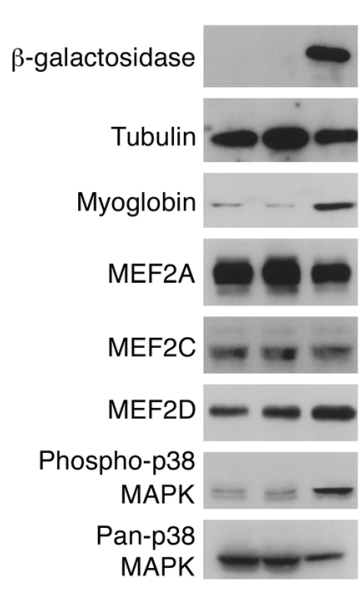

b

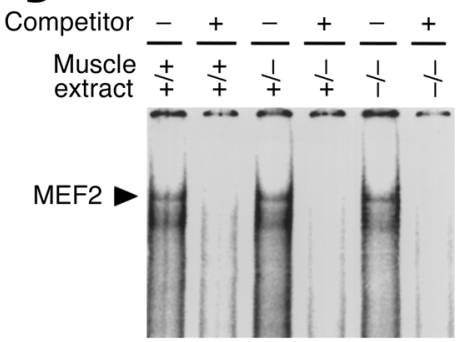

\section{Figure 3}

Immunoblots and assays for DNA-binding of MEF2 using WV muscle extracts from mice with wild-type $(+/+)$, heterozygous $(+/-)$, and homozygous (-/-) mutant CLCN1 alleles. (a) Western blot analyses of $\beta$-galactosidase, major MEF2 isoforms (MEF2A, $-2 \mathrm{C}$, and -2D), myoglobin, active form of p38 MAPK (phosphop38 MAPK), and total p38 MAPK. Tubulin blot showed equivalent loading of each lane. (b) Electrophoretic mobility shift assay of proteins extracted from $\mathrm{WV}$ muscles of different mice. The identity of the MEF2 protein-DNA complex (arrow) was confirmed by competition with 100-fold molar excess of unlabeled MEF2-binding oligonucleotides (competitor).

under the control of three copies of MEF2-binding elements derived from the desmin promoter (desMEF2lacZ) (27). The transgene expression is solely dependent on MEF2 transactivating function and provides readout for MEF2 activity that is suitable for physiological and pathological studies in intact animals.

Most skeletal muscles from adult desMEF2-lacZ mice have little lacZ expression (Figure 2), although abundant MEF2 proteins exist in these muscles. In mice carrying only one allele of the mutant CLCN1 gene (+/-), MEF2 was still kept inactive (Figure 2), consistent with the notion that the mutation of the CLCN1 gene in ADR myotonic mice is autosomal recessive. In mice homozygous for the CLCN1 mutation (-/-), MEF2 activity was dramatically upregulated in skeletal muscles (Figure 2). MEF2 activation was obvious in all the myotonic mice examined. Interestingly, MEF2 a significantly increased in white vastus (WV) muscles from ADR myotonic mice (-/-, homozygous for CLCN1 mutation), with a concomitant decrease in MHC IIb expression. Myoglobin, a marker for muscle oxidative capacity, also showed upregulation in myotonic muscles.

Marked induction of MEF2 transcriptional activity in myotonic skeletal muscles. Previously, we have shown that MEF2 is selectively active in slow/oxidative myofibers (21). Furthermore, endurance exercise and low-frequency stimulation, two protocols that can trigger transformation of glycolytic fibers into oxidative ones, enhance MEF2 activity in skeletal muscles (22). MEF2binding sites are necessary for fiber type-specific activities of myoglobin and TnI slow promoters, suggesting MEF2 plays a causative role in determining slow/oxidative fiber identity (21).

The observation that glycolytic fibers are converted into oxidative fibers in myotonic muscles prompted us to investigate whether MEF2 is involved in this pathological process. To this end, we introduced a lacZ reporter transgene into the ADR myotonic mice through conventional breeding of ADR myotonic mice with MEF2 indicator mice, which contain a lacZ transgene

\section{Figure 4}

Expression of HDAC isoforms in WV muscles from wild-type $(+/+)$, heterozygous (+/-), and homozygous (-/-) CLCN1 mutant mice. (a) Western blot analyses of HDAC1, -2 , and -3 (class I) and HDAC4, -5 , and -7 (class II) expression. (b) RNA transcripts of HDAC1-9 detected by RT-PCR. MITR is an alternative form of HDAC9.

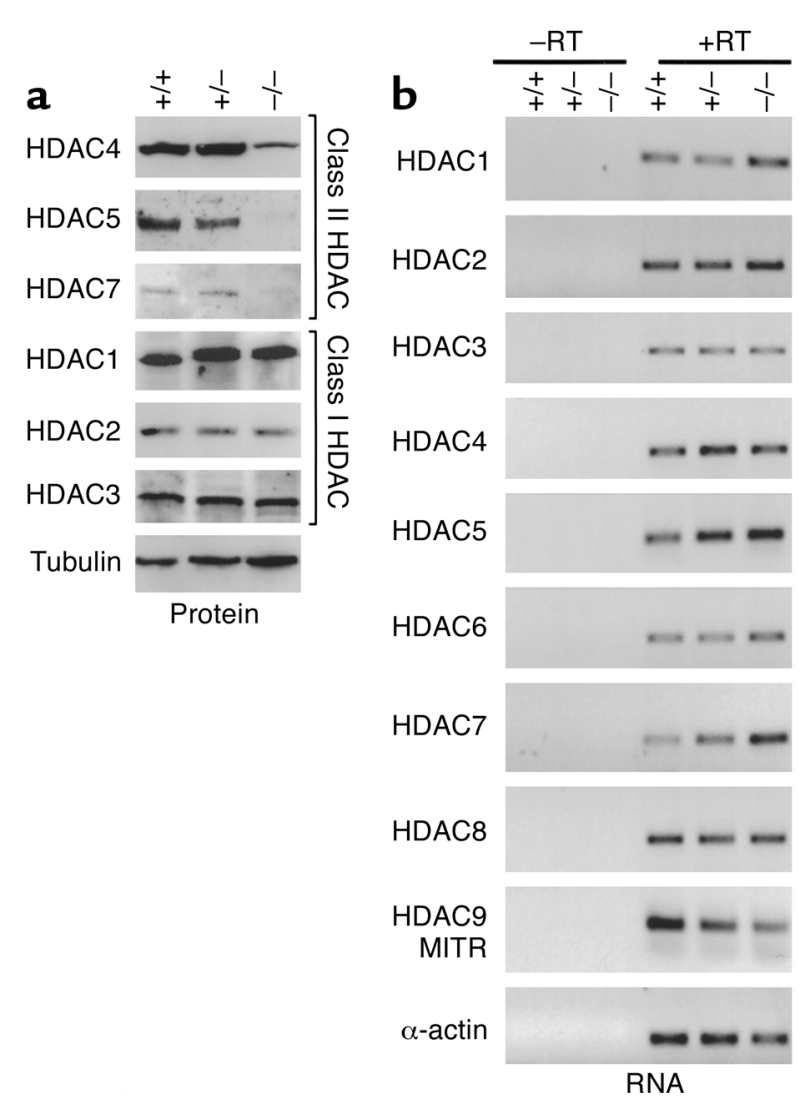


activation was limited to muscles composed mostly of fast glycolytic fibers (in wild-type mice), such as plantaris, extensor digitorum longus (EDL), and WV, but not in soleus, which has very few fast glycolytic fibers (34). MEF2 activation was observed in two strains of ADR myotonic mice: SWR/J-Clcn $1^{\text {adr-mto }}$ and $\mathrm{BALB} / \mathrm{cByJ}-\mathrm{Cl}$ cn $1^{\text {adr-mto }}$. The activation of MEF2 was monitored more quantitatively through immunoblotting with an $\mathrm{Ab}$ against $\beta$-galactosidase (Figure $3 \mathrm{a}$ ). The induction of MEF2 transcriptional activity was specific for skeletal muscles because no MEF2-dependent $\beta$-galactosidase expression was enhanced in other tissues examined, including heart and brain, where MEF2 expression level is high (data not shown). Previously, we reported that the MEF2-lacZ reporter was upregulated in skeletal muscles in response to exercise and chronic electrical stimulation (22). The level of lacZ expression in muscles from ADR mice greatly exceeded that seen in response to these stimuli.

Activation of MEF2 in myotonic muscles occurs without changes in MEF2 expression levels or MEF2 DNA-binding affinity. MEF2A, $-2 \mathrm{C}$, and $-2 \mathrm{D}$ are the major isoforms of MEF2 expressed in adult skeletal muscles. Their expression levels did not alter significantly in the WV muscles from myotonic mice compared with wildtype (+/+) or heterozygous CLCN1 mutant (+/-) littermates, as revealed upon Western blot analysis (Figure 3a). Myoglobin protein expression increased in myotonic WV muscles, indicative of enhancement of muscle oxidative capacity.

The p38 MAPK is a known activator for MEF2 (26, 35). Activated p38 MAPK phosphorylates conserved residues within the $\mathrm{C}$-terminal transactivating domain of MEF2 factors and thereby potentiates MEF2 transcriptional activity. We assayed for p38 activation in myotonic muscles by immunoblotting using an $\mathrm{Ab}$ recognizing the phospho-specific form of p38 MAPK, which is the activated form of this protein kinase. Indeed, p38 MAPK was activated in the WV muscles from myotonic mice (Figure 3a). The expression level of total p38 MAPK was not altered significantly in myotonic muscles, as assessed by immunoblot with a pan-p38 MAPK Ab (Figure 3a).

MEF2 is a bipartite transcription factor with an $\mathrm{N}$-terminal DNA-binding/dimerization domain and a C-terminal transactivating domain (36). One possible mechanism to regulate MEF2 activity is through modulating the DNA-binding affinity of MEF2. There were no significant changes in DNA binding of MEF2 proteins extracted from WV muscles of nonmyotonic and myotonic mice (Figure $3 \mathrm{~b}$ ), suggesting MEF2 DNA-binding affinity was not altered in myotonic muscles.

Class II HDAC deficiency in myotonic skeletal muscles. MEF2 transcriptional activity is repressed by class II HDAC complexes, which dock on the N-terminal DNA-binding domain of MEF2 and prevent MEF2 from activating its target genes (36-38). Class I HDACs (HDAC1, -2, -3, -8) are ubiquitously expressed, while class II HDACs (HDAC4, -5, -6, -7, -9) are enriched in muscle cells, where they interact with MEF2 through their long $\mathrm{N}$-terminal extensions (38). At the protein level, class II HDACs displayed a severe reduction in myotonic (-/-) WV muscles compared with wild-type $(+/+)$ and heterozygous $C L C N 1$ mutant $(+/-)$ littermates, while expression of the class I HDACs remained unchanged (Figure 4a). The decrease in class II HDAC expression seemed to occur after transcription, because no changes in HDAC RNA transcript levels were observed (Figure 4b). This downregulation of class II HDACs would be predicted to result in an increase in MEF2 transcriptional activities, as we observed in muscle fibers from ADR mice.

\section{Discussion}

Myofibers from animals are heterogeneous with respect to their metabolic, contractile, and morphological properties (30). Specialized myofiber phenotypes are highly plastic and can be altered under physiological and pathological circumstances (33). The dominant influence of motor neurons on fiber

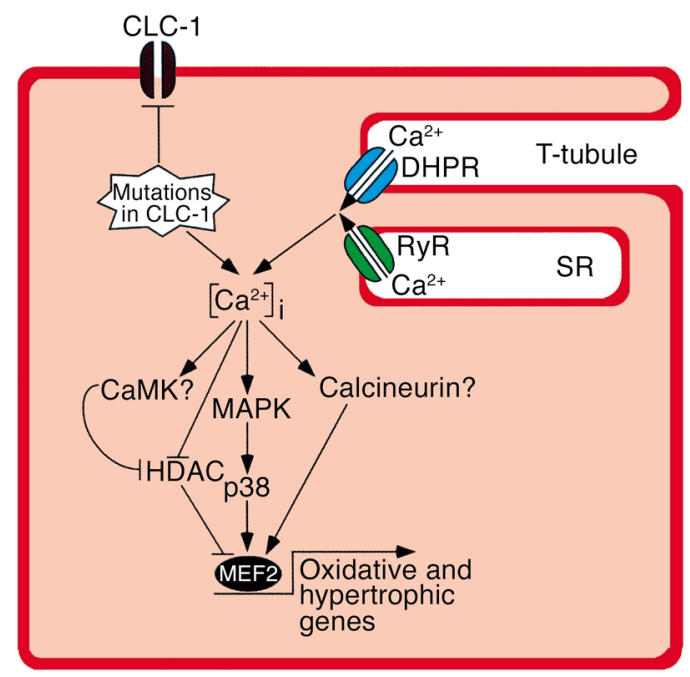

\section{Figure 5}

A model for MEF2 activation in myotonic muscles. CLC-1 chloride channels stabilize membrane potential in normal myofibers. In myotonic myofibers, membrane destabilization causes activation of the stress-responsive p38 MAPK, which is a known activator of MEF2. MEF2 activation in myotonic muscles also correlates with severe deficiency of class II HDACs, which function as repressors of MEF2-dependent gene expression. We propose that activated MEF2 is responsible for altered gene expression profiles in myotonic myofibers (shift toward oxidative phenotype and hypertrophy). Calcineurin and CaMK have previously been shown to activate MEF2. In vitro assays for calcineurin and CaMK showed no change of total enzymatic activities in myotonic muscles (data not shown); however, such assays do not measure potential differences in enzyme activation in vivo. Further in vivo experiments will define the roles of these signaling molecules in regulating gene expression in myotonic muscles. Other calcium-independent signaling pathways might also be responsible for the long-term changes of gene expression patterns in myotonic muscles. DHPR, dihydropyridine receptor; RyR, ryanodine receptor; T-tubule, transverse tubule; $\mathrm{SR}$, sarcoplasmic reticulum. 
type-specific gene expression is demonstrated by experiments in which cross-innervation or electrical pacing transforms one myofiber subtype into another $(39,40)$.

Previously, we have shown that MEF2 is required for slow/oxidative fiber-specific gene expression and is involved in transformation of fast/glycolytic myofibers into slow/oxidative ones $(21,22)$. In the present study, we demonstrated that the characteristic hyperexcitability of myotonic muscles led to dramatic upregulation of MEF2 transcriptional activity in the ADR mouse, which is a model for human myotonic disease. This upregulation of MEF2 would be expected to promote the sort of fiber-type transformation observed in ADR myotonic mice. The pathological hyperactivity of myotonic muscles mimics the effects of endurance exercise and low-frequency stimulation, both of which activate MEF2 and switch fast/glycolytic fibers into slow/oxidative ones (22).

Activation of MEF2 in myotonic mice is restricted to skeletal muscles composed primarily of fast glycolytic fibers (before fiber-type transformation), such as PLA, EDL, and WV muscles. Soleus muscles, which have very few fast glycolytic fibers, showed little induction of MEF2 activity. In normal mice, the soleus functions as an antigravity muscle and contracts almost constantly to maintain posture of the animal (41). Hyperexcitability of the sarcolemma due to the loss of chloride channel functions has a minimal effect in soleus muscles since they receive neural input and contract almost all the time. Fast glycolytic fibers are only employed during occasional powerful movements and remain quiescent otherwise (41). They experience the least amount of muscle activity in normal mice, and chloride channel mutations will have the strongest effect on these fibers.

MEF2 activity is regulated by many signaling pathways, among which, MAPKs have been shown to act directly on specific residues of MEF2A, -C, and -D, thereby augmenting MEF2 transactivating functions (42). MEF2 also integrates signals generated from calcium-dependent enzymes, such as calcineurin and CaMKs $(20,21)$. In skeletal muscle cells, calcineurin potentiates MEF2 transcriptional activity in a manner independent of effects on MEF2 DNA binding (22). MEF2 proteins can also function as transcriptional repressors through their association with class II HDACs (36). Activated CaMKs relieve this form of repression by dissociating HDAC from MEF2 and promoting the export of HDAC out of the nucleus (23). The net effect is upregulation of MEF2-dependent gene transcription. Here, we observed that the expression level of class II HDAC proteins was significantly reduced in skeletal muscles from myotonic mice compared with wild-type littermates. The severe deficiency (>20-fold decrease) of class II HDACs would be predicted to de-repress MEF2 activity. The activation of p38 MAPK would further augment MEF2 function. The mechanisms by which class II HDACs are selectively downregulated in myotonic muscles remain to be elucidated.
How does membrane destabilization resulting from chloride channel mutations lead to MEF2 activation and fiber-type transformation in myotonic muscles? One partial explanation is that stress experienced by myotonic myofibers can activate p38 MAPK, which subsequently enhances MEF2 transcriptional activity (Figure 5). Perturbed calcium homeostasis might be responsible for the long-term cellular changes in myotonic muscles. Calcium/calmodulin-dependent signaling molecules, such as calcineurin, have been implicated in regulating MEF2 activities and modulating fiber-type identities (21). Calcineurin is also involved in skeletal muscle hypertrophy (43-45), which is another clinical feature of most patients suffering myotonia congenita (16). MEF2 can act as a transcriptional effector of calcineurin signaling in both fibertype transformation and muscle hypertrophy $(20,22)$. Activation of MEF2, as we observed in myotonic muscles, might explain the adaptations of the affected skeletal muscles in myotonia patients (Figure 5).

The use of pharmacological agents that can block calcium channels and/or antagonize calcium-dependent enzymes (e.g., calcineurin and CaMKs) will help to determine whether calcium plays critical roles in the development of clinical features associated with myotonia congenita. These same agents might be used in combination with drugs currently available for the treatment of myotonia patients.

\section{Acknowledgments}

We thank J. Shelton for his help with histochemistry and A. Tizenor and J. Page for assistance with the manuscript. This work was supported by grants from the NIH, the D.W. Reynolds Clinical Cardiovascular Research Center, the Texas Advanced Technology Program and a grant from the Muscular Dystrophy Association to E.N. Olson.

\footnotetext{
1. Barchi, R.L. 1997. Ion channel mutations and diseases of skeletal muscle. Neurobiol. Dis. 4:254-264.

2. England, J.D. 2001. Mutant sodium channels, myotonia, and propofol. Muscle Nerve. 24:713-715.

3. Lehmann-Horn, F., Mailander, V., Heine, R., and George, A.L. 1995 Myotonia levior is a chloride channel disorder. Hum. Mol. Genet. 4:1397-1402.

4. Ptacek, L.J., Johnson, K.J., and Griggs, R.C. 1993. Genetics and physiology of the myotonic muscle disorders. N. Engl. J. Med. 328:482-489.

5. Barchi, R.L. 1998. Phenotype and genotype in the myotonic disorders. Muscle Nerve. 21:1119-1121.

6. Steinmeyer, K., Ortland, C., and Jentsch, T.J. 1991. Primary structure and functional expression of a developmentally regulated skeletal muscle chloride channel. Nature. 354:301-304.

7. Fahlke, C., Rhodes, T.H., Desai, R.R., and George, A.L., Jr. 1998. Pore stoichiometry of a voltage-gated chloride channel. Nature. 394:687-690.

8. Koch, M.C., et al. 1993. Evidence for genetic homogeneity in autosomal recessive generalised myotonia (Becker). J. Med. Genet. 30:914-917.

9. Kubisch, C., Schmidt-Rose, T., Fontaine, B., Bretag, A.H., and Jentsch, T.J. 1998. CLC-1 chloride channel mutations in myotonia congenita: variable penetrance of mutations shifting the voltage dependence. Hum. Mol. Genet. 7:1753-1760.

10. Maduke, M., Miller, C., and Mindell, J.A. 2000. A decade of CLC chloride channels: structure, mechanism, and many unsettled questions. Annu. Rev. Biophys. Biomol. Struct. 29:411-438.

11. Waldegger, S., and Jentsch, T.J. 2000. From tonus to tonicity: physiology of CLC chloride channels. J. Am. Soc. Nephrol. 11:1331-1339.

12. Steinmeyer, K., et al. 1991. Inactivation of muscle chloride channel by transposon insertion in myotonic mice. Nature. 354:304-308.
} 
13. Jockusch, H. 1990. Allelic mutations at the myotonia locus adr. Mouse Genome. 87:72.

14. Gronemeier, M., et al. 1994. Nonsense and missense mutations in the muscular chloride channel gene Clc-1 of myotonic mice. J. Biol. Chem. 269:5963-5967.

15. Reininghaus, J., Fuchtbauer, E.M., Bertram, K., and Jockusch, H. 1988. The myotonic mouse mutant ADR: physiological and histochemical properties of muscle. Muscle Nerve. 11:433-439.

16. Sun, C., Tranebjaerg, L., Torbergsen, T., Holmgren, G., and Van Ghelue, M. 2001. Spectrum of CLCN1 mutations in patients with myotonia congenita in Northern Scandinavia. Eur. J. Hum. Genet. 9:903-909.

17. Crews, J., Kaiser, K.K., and Brooke, M.H. 1976. Muscle pathology of myotonia congenita. J. Neurol. Sci. 28:449-457.

18. Naya, F.S., and Olson, E.N. 1999. MEF2: a transcriptional target for signaling pathways controlling skeletal muscle growth and differentiation. Curr. Opin. Cell Biol. 11:683-688.

19. Olson, E.N., and Williams, R.S. 2000. Calcineurin signaling and muscle remodeling. Cell. 101:689-692.

20. Passier, R., et al. 2000. CaM kinase signaling induces cardiac hypertrophy and activates the MEF2 transcription factor in vivo. J. Clin. Invest. 105:1395-1406.

21. Wu, H., et al. 2000. MEF2 responds to multiple calcium-regulated signals in the control of skeletal muscle fiber type. EMBO J. 19:1963-1973.

22. Wu, H., et al. 2001. Activation of MEF2 by muscle activity is mediated through a calcineurin-dependent pathway. EMBOJ. 20:6414-6423.

23. McKinsey, T.A., Zhang, C.L., Lu, J., and Olson, E.N. 2000. Signal-dependent nuclear export of a histone deacetylase regulates muscle differentiation. Nature. 408:106-111.

24. McKinsey, T.A., Zhang, C.L., Olson, E.N. 2000. Activation of the myocyte enhancer factor- 2 transcription factor by calcium/calmodulin-dependent protein kinase-stimulated binding of 14-3-3 to histone deacetylase 5. Proc. Natl. Acad. Sci. USA. 97:14400-14405.

25. Kato, Y., et al. 1997. BMK1/ERK5 regulates serum-induced early gene expression through transcription factor MEF2C. EMBOJ. 16:7054-7066.

26. Zhao, M., et al. 1999. Regulation of the MEF2 family of transcription factors by p38. Mol. Cell. Biol. 19:21-30.

27. Naya, F.J., Wu, C., Richardson, J.A., Overbeek, P., and Olson, E.N. 1999. Transcriptional activity of MEF2 during mouse embryogenesis monitored with a MEF2-dependent transgene. Development. 126:2045-2052.

28. Gossett, L.A., Kelvin, D.J., Sternberg, E.A., and Olson, E.N. 1989. A new myocyte-specific enhancer-binding factor that recognizes a conserved element associated with multiple muscle-specific genes. Mol. Cell. Biol. 9:5022-5033.

29. Ogilvie, R.W., and Feeback, D.L. 1990. A metachromatic dye-ATPase method for the simultaneous identification of skeletal muscle fiber types I, IIA, IIB and IIC. Stain Technol. 65:231-241.
30. Booth, F.W., and Baldwin, K.M. 1996. Muscle plasticity: energy demand and supply processes. In The handbook of physiology: integration of motor, circulatory, respiratory and metabolic control during exercise. L. B. Bowell and J.T. Shepard, editors. American Physiological Society. Bethesda, Maryland, USA. $1075-1123$.

31. Schiaffino, S., and Reggiani, C. 1996. Molecular diversity of myofibrillar proteins - gene regulation and functional significance. Physiol. Rev. 76:371-423.

32. Weiss, A., and Leinwand, L.A. 1996. The mammalian myosin heavy chain gene family. Annu. Rev. Cell Dev. Biol. 12:417-439.

33. Pette, D., and Staron, R.S. 2001. Transitions of muscle fiber phenotypic profiles. Histochem. Cell. Biol. 115:359-372.

34. Ovalle, W.K., Bressler, B.H., Jasch, L.G., and Slonecker, C.E. 1983. Abnormal distribution of fiber types in the slow-twitch soleus muscle of the C57BL/6J dy2J/dy2J dystrophic mouse during postnatal development. Am. J. Anat. 168:291-304.

35. Yang, S.H., Galanis, A., and Sharrocks, A.D. 1999. Targeting of p38 mitogen-activated protein kinases to MEF2 transcription factors. Mol. Cell. Biol. 19:4028-4038.

36. Lu, J., McKinsey, T.A., Nicol, R.L., and Olson, E.N. 2000. Signal-dependent activation of the MEF2 transcription factor by dissociation from histone deacetylases. Proc. Natl. Acad. Sci. USA. 97:4070-4075.

37. Miska, E.A., et al. 1999. HDAC4 deacetylase associates with and represses the MEF2 transcription factor. EMBOJ. 18:5099-5107.

38. Lu, J., McKinsey, T.A., Zhang, C.L., and Olson, E.N. 2000. Regulation of skeletal myogenesis by association of the MEF2 transcription factor with class II histone deacetylases. Mol. Cell. 6:233-244.

39. Buller, A.J., Eccles, J.C., and Eccles, R.M. 1960. Differentiation of fast and slow muscles in the cat hind limb. J. Physiol. (London). 150:399-416.

40. Pette, D., and Vrbova, G. 1992. Adaptation of mammalian skeletal muscle fibers to chronic electrical stimulation. Rev. Physiol. Biochem. Pharmacol. 120:115-202.

41. Hennig, R., and Lomo, T. 1985. Firing patterns of motor units in normal rats. Nature. 314:164-166.

42. Han, J., and Molkentin, J.D. 2000. Regulation of MEF2 by p38 MAPK and its implication in cardiomyocyte biology. Trends Cardiovasc. Med. 10:19-22

43. McKinsey, T.A., Zhang, C.L., and Olson, E.N. 2002. MEF2: a calciumdependent regulator of cell division, differentiation and death. Trends Biochem. Sci. 27:40-47.

44. Musaro, A., McCullagh, K.J., Naya, F.J., Olson, E.N., and Rosenthal, N. 1999. IGF-1 induces skeletal myocyte hypertrophy through calcineurin in association with GATA-2 and NF-ATc1. Nature. 400:581-585

45. Semsarian, C., et al. 1999. Skeletal muscle hypertrophy is mediated by a Ca2+-dependent calcineurin signalling pathway. Nature. 400:576-581. 